\title{
In silico comparative study of SARS-CoV-2 proteins and antigenic proteins in BCG, OPV, MMR and other vaccines: evidence of a possible putative protective effect
}

\author{
Sondes Haddad-Boubaker ( $\sim$ sondeshaddadboubaker@gmail.com ) \\ Institut Pasteur de Tunis, Tunis, Tunisia https://orcid.org/0000-0001-9705-4387 \\ Houcemeddine Othman \\ University of the Witwatersrand, Johannesburg, South Africa \\ Rabeb Touati \\ Faculty of Medicine of Tunis, University of Tunis El Manar, Tunisia \\ Kaouther Ayouni \\ Institut Pasteur de Tunis, Tunis, Tunisia \\ Marwa Lakhal \\ Faculty of Medicine of Tunis, University of Tunis El Manar, Tunisia \\ Imen Ben Mustapha \\ Institut Pasteur de Tunis, Tunis, Tunisia \\ Kais Ghedira \\ Institut Pasteur de Tunis, Tunis, Tunisia \\ Maher Kharrat \\ Faculty of Medicine of Tunis, University of Tunis El Manar, Tunisia \\ Henda Triki \\ Institut Pasteur de Tunis, Tunis, Tunisia
}

\section{Research Article}

Keywords: SARS-CoV-2, BCG, OPV, MMR, immunogenicity, Vaccine, Putative protection

Posted Date: January 21st, 2021

DOI: https://doi.org/10.21203/rs.3.rs-105598/v3

License: @ (i) This work is licensed under a Creative Commons Attribution 4.0 International License. Read Full License 


\section{Abstract}

Background. Coronavirus Disease 2019 (COVID-19) is a viral pandemic disease that may induce severe pneumonia in humans. In this paper, we investigated the putative implication of 12 vaccines, including BCG, OPV and MMR in the protection against COVID-19. Sequences of the main antigenic proteins in the investigated vaccines and SARS-CoV-2 proteins were compared to identify similar patterns. The immunogenic effect of identified segments was, then, assessed using a combination of structural and antigenicity prediction tools.

Results. A total of 14 highly similar segments were identified in the investigated vaccines. Structural and antigenicity prediction analysis showed that, among the identified patterns, three segments in Hepatitis B, Tetanus, and Measles proteins presented antigenic properties that can induce putative protective effect against COVID-19.

Conclusions. Our results suggest a possible protective effect of HBV, Tetanus and Measles vaccines against COVID-19, which may explain the variation of the disease severity among regions.

\section{Background}

Since December 2019, an emerging coronavirus called Severe Acute Respiratory Syndrome Coronavirus 2 (SARS-CoV-2) has been spreading worldwide. This novel pathogen is responsible for the Coronavirus Disease 2019 (COVID-19), causing a worldwide pandemic, as declared by the World Health Organization (WHO) in March 2020 [1]. So far, SARS-CoV-2 has been responsible for more than 91 million confirmed cases and more than a million fatalities (from December 8, 2019 to January 15, 2021) [2].

SARS-CoV-2 is an enveloped, positive-sense single-stranded RNA virus. It belongs to the family of Coronaviridae, the subfamily of Orthocoronavirinae and the genus Betacoronavirus [3]. The viral genome is composed of approximately 29903 nucleotides; it contains two untranslated regions ( $5^{\prime}$ and $3^{\prime}$ ) and eleven Open Reading Frames (ORF) encoding twelve proteins including the Spike (S) and the Nucleocapsid $(\mathrm{N})$ proteins identified as the main antigenic proteins $[4,5]$.

The number of COVID-19 patients and death cases varied from a region to another [6]. In many countries, an important number of confirmed cases were reported, such as in the United States of America (USA), Brazil, India and Russia causing [6-9]. For instance, in the USA, more than eight million confirmed cases and two hundred thousand deaths were recorded until the $18^{\text {th }}$ of October $2020[6,9]$. However, in other regions, such as Madagascar, Sierra Leone, Nicaragua and Uruguay, the number of cases seems to be limited [6, 10, 11] and no more than three hundred deaths were notified [6]. The variation in the number of deaths and infections among countries can be explained by different factors, such as health infrastructure, mitigation strategies and also cultural behavior [12, 13]. The immunological background of the population, mainly due to the vaccination strategies used in those countries was also suggested [1315]. Indeed, it was previously demonstrated that administration of attenuated vaccines such as OPV (Oral Poliovirus Vaccine), MMR (Measles, Mumps and Rubella vaccines) and BCG (Bacillus Calmette-Guérin) vaccines could improve the innate immune response to fight different pathogens [13-15]. Furthermore, it was suggested that the adoption of a universal and long-standing BCG policy may have a protective effect against COVID-19 [13]. However, and to date, no comprehensive fundamental evidence showed a relationship between regular vaccination and the acquisition of immunity to SARS-CoV-2. In recent epidemiological study, based on a large cohort of patients, no links between the administration of BCG vaccine and COVID-19 severity was found [16]; but, after refining the epidemiological study, a strong correlation was reported [17]. The protective potential of the MMR vaccine was also investigated based on bioinformatic analysis of the S protein [18]. However, no similarity with the crystal structure of S protein, in the Wuhan-Hu-1 isolate (MN908947.3), has been reported [18].

In this paper, we investigated the putative protective role against COVID-19 of three live attenuated vaccines (BCG, OPV and MMR) and nine inactivated vaccines (Tetanus, Corynebacterium diphtheriae, Bordetella pertussis, Hepatitis B, Hepatitis A, Haemophilus influenzae type $B$ (Hib) and Streptococcus pneumoniae vaccines (PCV10)). Our aim was to identify similar amino-acid patterns in all SARS-CoV-2 proteins and the main antigenic proteins of the above-mentioned vaccines and to predict their immunogenicity, using a combination of bioinformatic tools. The in silico identified patterns may be the target of cross-reactive antibodies against their specific pathogen and SARS-CoV-2 and/or may induce cellular immunity.

\section{Results}

\section{Amino acid sequence alignment and hot spot analysis}


The global amino acid identity between the main antigenic protein of investigated vaccines and SARS-CoV-2 proteins does not exceed $63 \%$. For structural proteins, it varied between $21 \%$ and $55 \%$ (identity levels for the S and M proteins respectively with the Polyprotein E1/E2 of the Rubella virus and the HAV VP1 protein). For non-structural proteins, identity levels varied between 21 and $63 \%$ (identity rates of ORF1a and ORF3a proteins respectively with HBsAg-adr protein of Hepatitis B virus and Tetanus Toxin protein) (Supplementary material 1).

Similar segments with main vaccine antigenic proteins were identified along with structural and non-structural proteins of SARS-CoV-2. The majority were shorter than five consecutive amino-acids for all SARS-CoV-2 proteins (Supplementary material 2-13). Nevertheless, a total of twelve patterns of six to eight similar consecutive amino-acids were identified in comparison with the main antigenic proteins of Poliovirus, Measles, Streptococcus pneumoniae, Tetanus, Mumps, Hepatitis B, Hib and BCG vaccines (Table1). Two similar segments were identified through comparison of Poliovirus, Measles, PCV10 and Hib proteins and SARS-CoV-2 structural proteins (S and N) and also non-structural proteins (ORF 1a, ORF 6 and ORF 8). In contrast, Tetanus, Mumps, Hepatitis B and BCG antigenic proteins showed no more than one similar segment with SARS-CoV-2 proteins (Table1). Among the described peptides, seven were similar to others in the S protein of SARS-CoV-2 and were identified in the antigenic proteins in poliovirus Sabin 3, S pneumoniae, tetanus, Mumps, Hepatitis B and Hib vaccines. The pattern's length varied between six and seven amino acids. Also, one peptide of eight amino acids (GTSPARMA), detected in the Poliovirus VP1 sequence, matched with the N protein of the SARS-CoV-2.

We also identified two discontinuous patterns of 10 amino-acids each, DISGFNSSVI and MSLSLLDLYL, in the tetanus toxin and the hemagglutinin Measles virus proteins which had 90\% and 80\% similarity with matching segments, DISGINASVV (1168-1177aa), IELSLIDFYL (2-11aa), in the S and ORF7b proteins of SARS-CoV-2 respectively.

Table1: Description of similar patterns of more than five amino-acids obtained in vaccine antigenic proteins and SARS-CoV-2 proteins

\begin{tabular}{|c|c|c|c|c|c|c|c|}
\hline \multirow[t]{2}{*}{ Vaccine } & \multirow{2}{*}{$\begin{array}{l}N^{\circ} \text { of } \\
\text { similar } \\
\text { segment }\end{array}$} & \multicolumn{3}{|l|}{ Vaccine protein } & \multicolumn{3}{|c|}{ SARS-CoV-2 Protein } \\
\hline & & Designation & Segment & Position & Designation & Segment & Position \\
\hline \multirow[t]{2}{*}{ Poliovirus } & \multirow[t]{2}{*}{2} & VP1 protein (Sabin 1) & GTAPARIS & 188-195 & $\mathrm{N}$ & GTSPARMA & 203-211 \\
\hline & & VP1 protein (Sabin 3) & LDPLSE & 289-295 & S & LDPLSE & 293-299 \\
\hline \multirow[t]{2}{*}{ Measles } & \multirow[t]{2}{*}{2} & \multirow[t]{2}{*}{ Fusion protein } & QECLRG & $359-364$ & ORF6 & QECVRG & $21-27$ \\
\hline & & & IQVGSRR & $433-440$ & ORF8 & IRVGARK & $47-54$ \\
\hline \multirow[t]{2}{*}{$\begin{array}{l}\text { Streptococcus } \\
\text { pneumoniae }\end{array}$} & \multirow[t]{2}{*}{2} & $\begin{array}{l}\text { Capsular polysaccharide } \\
\text { biosynthesis protein } \\
\text { (serotype19F, 18C, 14, 7, 4, 1) }\end{array}$ & IGFLAGVI & $182-190$ & $S$ & LGFIAGLI & $\begin{array}{l}1218- \\
1226\end{array}$ \\
\hline & & $\begin{array}{l}\text { Capsular polysaccharide } \\
\text { biosynthesis protein } \\
\text { (serotype19F, 18C, 14, 5) }\end{array}$ & SSVAFA & $33-39$ & $S$ & NSVAYS & 703-708 \\
\hline \multirow[t]{2}{*}{ Hib } & \multirow[t]{2}{*}{2} & \multirow[t]{2}{*}{$\begin{array}{l}\text { Capsular polysaccharide } \\
\text { biosynthesis protein }\end{array}$} & KNINDS & $210-215$ & S & KNLNES & $\begin{array}{l}1191- \\
1196\end{array}$ \\
\hline & & & FILNKKI & $73-79$ & ORF1a & FLLNKEM & $\begin{array}{l}3183- \\
3189\end{array}$ \\
\hline BCG & 1 & Immunogenic protein MPB64 & IFMLVT & $5-11$ & E & VFLLVT & $25-31$ \\
\hline Tetanus & 1 & Toxin protein & NILMQY & $84-90$ & S & NLLLQY & $751-756$ \\
\hline Mumps virus & 1 & Fusion protein & DISTEL & $448-454$ & $S$ & DISTEI & $467-473$ \\
\hline Hepatitis B & 1 & HBs Ag-adr & PGTSTTS & $111-117$ & S & PGTNTSN & $600-606$ \\
\hline
\end{tabular}

\section{Immunogenicity prediction}

First, we focused on characterizing the immunogenicity of the matching sequences with $\mathrm{S}$ and $\mathrm{N}$ proteins for their involvement in modulating the immune response of the host $[19,20]$. 
Regarding the pattern GTAPARIS matching with $\mathrm{N}$ protein sequence (GTSPARMA), it did not map to the structure of the $\mathrm{N}$ protein from SARS-CoV-2. Moreover, no significant match with $\mathrm{CMH}-\mathrm{I}$ predicted epitope was distinguished. The prediction of the B-cell epitope using the $\mathrm{N}$ protein sequence showed a potential antigenic peptide of 51 amino acids (165-216) that harbors the pattern GTSPARMA identified from our similarity search.

Among the seven patterns identified in the SARS-CoV-2 S protein, four segments (LDPLSE, NSVAYS, NLLLQY, PGTNTSN) from Polio, PCV10, Tetanus and HBV vaccines, respectively, have been mapped on the structure of the spike protein S1 subunit (Figure 1A). We were also able to map one other pattern, KNLNE, on the structure of the six-helical bundle fusion core solved independently (S2 subunit) from the rest of the ectodomain. The two other patterns (LGFIAGLI, and DISTEI) were not solved by the electron density map from the Cryo-EM structures. Among the five retained patterns, the segments PGTNTSN and LGFIAGLI showed a putative interaction with one of the MHC-I receptors predicted by IEDB analysis resource NetMHCpan. Furthermore, the prediction for these two peptides showed a weak peptide score of 0.07 and 0.02 , respectively ( 0 indicates no MHC-I capacity, and 1 indicates a high probability). The segment PGTNTSN, existing in the $\mathrm{Hbs}$ Ag of Hepatitis B virus adr strain, is located in a turn region.

On the other hand, the prediction of epitopes for B-cell response using Bepipred 2.0 from the IEDB analysis resource showed the implication of four putative patterns from the total set of the seven segments, namely LDPLSE, NSVAYS, DISTEI and PGTNTSN. These segments match the predicted epitopes LDPL, YTMSLGAENSVAYSNN, NLDSKVGGNYNYLYRLFRKSNLKPFERDISTEIYQAGSTPCNGVEGFNCYFPLQSYGFQPTN and TNTSN (Figure 1 B). The sequence KNLNES does not fall in a putative B-cell epitope region.

We also calculated the Solvent Accessible Surface Area (SASA) using different probe radii to allow better insight into the possible interaction of antibody Complementarity-Determining Regions (CDRs) to the predicted epitopes (Figure 1C). Our results show that exposure to both water molecules and the antibody paratope is only preserved for the segment "PGTNTSN". Consequently, the SASA values at probe radii of $1.4 \AA, 5 \AA$, and $10 \AA$ are $528.69 \AA^{2}, 497.6 \AA^{2}$, and $305.38 \AA^{2}$, respectively.

Second, we focused on a list of hits that belonged to the investigated vaccine sequences and that match any of the other proteins of SARS-CoV-2. All the patterns have been explored for their antigenic potential using IEDB Bepipred and IEDB NetMHCpan methods. None of the investigated patterns showed a significant putative B-cell antibody binding property. Discontinuous patterns with more than ten residues were discarded from the analysis as they showed low levels of similarity. Consequently, we have retained two segments from Tetanus toxin protein (DISGFNSSVI) and chain A hemagglutinin protein of the Measles virus (MSLSLLDLYL) that significantly matched SARS-CoV-2 Spike and ORF7b proteins, respectively. The segment DISGINASVV of the S protein (Figure 2A) showed a putative interaction with the MHC-I receptor encoded by one of the corresponding HLA alleles. DISGINASVV and corresponding matching segment DISGFNSSVI showed high peptide scores of 0.88 and 0.76 for the SARS-CoV-2 S and the tetanus toxin proteins, respectively. The segment DISGINASVV is part of the six-helical bundle fusion core of the spike protein. It belongs to the HR2 domain as a random coil structure [21]. The peptide shows an extended conformation within its native environment stabilized by the residues of a small groove formed between two HR1 parallel helices from different monomers. The SASA value for DISGINASVV peptide is $504.88 \AA^{2}$. In contrast, its matching sequence from Tetanus toxin DISGFNSSVI corresponds to a SASA value of $243.3 \AA^{2}$ (Figure 2B) and the Bepipred tool shows only a partial implication of the sub-string "DISGI" as an epitope in the context of B-cell response.

Regarding the ORF7b and Measles hemagglutinin proteins, the identified similar segments overlap significantly with regions of putative T-cell antigenicity. The matching segment of the Measles hemagglutinin protein (Figure 2C) corresponded to a random coil segment (MSLS) spanned by an alpha helix of six residues (LLDLYL) in the crystal structure of the hemagglutinin [22]. The segment also interacts with a large pocket formed mainly by four strands of a beta-sheet containing many aromatic amino acids. The pocket is similar to the groove of the MHC-I molecule (Figure $2 \mathrm{C}$ and supplementary material 14). Moreover, MSLSLLDLYL corresponds to a SASA measured at $439.19 \AA^{2}$ (Figure 2B). The NetMHCpan tool predicted an antigenicity score of 0.18 for the MSLSLLDLYL segment using the sequence of ORF7b. We also noticed that the matching segment of the Measles hemagglutinin Protein, i.e "IELSLIDFYL" is represented by a substring "IELSLIDFY" that shows the highest antigenicity score of 0.59 among all the predicted epitopes.

\section{Discussion}

In this study, we investigated the potential protective effect against COVID-19 induced by regularly used vaccines. In the aim to assess their possible implication of in the immune response against SARS-CoV-2, we used a combination of sequence similarity analysis, 
structural and antigenicity prediction tools to evaluate main antigenic proteins in twelve commonly used vaccines including BCG, OPV and MMR vaccines.

In our study, we identified of similar patterns and found that most of the detected segments were shorter than five amino acids; therefore, they could not constitute a putative T-cell or B-cell epitopes [23-25].

Nevertheless, twelve patterns of six to eight amino-acids were found and further investigated. We think that PGTNTSN is the most putative to bind to endogenous antibodies among the four patterns that have been identified by the B-cell epitope prediction tool. Segments of less than 5 amino acids such as the LDPL, a substring of the LDPLSE, are rarely responsible for inducing humoral immunity response [25]. Moreover, NSVAYS and DISTEI segments are shorter with 10 and 56 amino acids less than the matching predicted epitopes using the entire sequence of the spike protein from SARS-CoV-2. In such a case, the sequence length would be a constraining factor in reproducing the immunological properties for the studied vaccines. That also applies to GTSPARMA segment which is a substring of 51 amino acid putative epitope from the N protein. On the other hand, the PGTNTSN segment of SARS-CoV-2 matches with the predicted epitope TNTSN which is only shorter by two amino acids, compared to both patterns identified for SARSCoV-2 and its matching segment on HBs Ag-adr.

The pattern PGTNTSN detected in HBsAg of Hepatitis B virus corresponded to an exposed site in the S protein and showed the highest values of accessible surface area compared to the segments identified in the S1 subunit. Additionally, the accessibility of PGTNTSN to the probing spheres mimicking the CDRs antibodies supports its implication in the B-cell mediated response. Thus, its structural properties were consistent with its putative neutralizing capacity. Naturally, the antibodies would be able to recognize the targeted epitope on the whole assembled structure of the virus, and therefore, the epitope must be accessible at the surface of the spike protein. On the other hand, in their recent attempt to establish the antigenicity map of SARS-CoV-2, Zhang et al (2020) have found that a segment called IDh spanning residues 522-646 induces a positive B-cells reaction in sera of convalescent COVID-19 patients [20]. The pattern PGTNTSN was included in the IDh epitope and we were able to identify strong prediction metrics using the IEDB Bepipred tool. Therefore, the induced immunological reaction by this segment would be a humoral response. Furthermore, our results were in agreement with the work published by Tajiri et al, [26] who showed that two regions of HBsAg (residues 104-123 and 108-123) containing the epitope matching the PGTNTSN segment of SARS-CoV-2, were able to bind with two human monoclonal antibodies. This highlighted the immunogenic capability of these segments. There have been concerns about the antibody-dependent enhancement (ADE) of the SARS-CoV-2 infection due to the possible activation of effector functions [27]. The antibody repertoire is thought to be the main culprit for such an effect [28]. However, its magnitude still unknown and recent evidence suggests a nonsignificant or unclear contribution in enhancing the infectivity of SARS-CoV-2. For instance, the expression of Fcy receptors through which the effector functions are triggered seems to be very low in alveolar, bronchial, and nasal-cavity epithelial cells (idem). Moreover, it is difficult to distinguish the contribution of the antibody-dependent enhancement of the infection from a severity due to other factors. Recently, in a detailed review, Arvin et al have stated that current clinical experience is insufficient to implicate a role for ADE of disease, or immune enhancement by any other mechanism, in the severity of COVID-19 [28].

The segment PGTNTSN is located away from the RBD interaction site to ACE2, separated by an approximate distance of $75 \AA$. However, the putative antigen, is very close to the fusion peptide SFIEDLLFNKV (residues 816-826 on the PDB structure 7BYR) located at an approximate distance of $35 \AA$. Moreover, the same region includes the S21P2 segment that has been identified as the epitope for antibodies targeting protein $S$ and enabling the neutralization of the SARS-CoV-2 pseudovirus infection [28]. Therefore, it would be possible to have the same scenario for the PGTNTSN predicted epitope. Furthermore, the location of the PGTNTSN segment overlaps with a putative interaction surface with TMPRSS2 which would impact the cleavage of S1/S2 and S2 sites required for the priming of the S protein $[29,30]$.

On the other hand, and considering the S protein conservation, which is constantly facing a selective pressure from the immune system, several studies demonstrated the existence of highly conserved domains in the S protein such as "SD2.1" (amino acids 589-605) which matches with the 'PGTNTSN' segment (600-606) [31-33]. Still, only, randomized controlled trials might provide evidence of induced protective effect against COVID-19. In many countries, the HBV vaccine is commonly recommended or mandatory for healthcare and wet lab workers. Therefore, it would be interesting to investigate the prevalence of SARS-CoV-2 and clinical manifestations of COVID-19 among HBV vaccinated health workers.

Interestingly, our analysis showed the presence of two segments of ten amino acids from the Tetanus toxin protein and the chain A of the Measles hemagglutinin protein, similar to others located in the S and ORF7b proteins of SARS-CoV-2. The segment DISGINASVV, matching with the toxin tetanus protein has been previously described to be part of an antigenic peptide in the S protein of SARS-CoV-2 
[34]. Trigueiro-Louro et al. performed a structure-based strategy targeting highly conserved regions in the Spike domains and demonstrated that the domain "CD-HR2.1" (amino acids 1112-1232), that matches with the regions DISGINASVV, is a "highly conserved druggable regions" [14]. Regarding the segment matching with the ORF7b protein, which may have an accessory function and whose role is yet to be determined [35], we could not exclude its possible immunogenic role. On the other hand, we have also recorded a significant global identity level between the Measles fusion and hemagglutinin proteins and SARS-CoV-2 spike, envelope and matrix proteins (45-50\%) (suppl mat. 1). Furthermore, another study using other Measles and Rubella sequences, different from Edmonston Measles and Wistar RA 27/3 Rubella vaccine strains, revealed similarity between the N terminal region of SARS-COV-2 Spike protein and the Fusion protein of Measles virus as well as the envelope protein of Rubella virus. Still, no similarity was obtained with the crystal structure [18]. It was previously demonstrated that live attenuated vaccines such as OPV, BCG and MMR could improve the innate immune response to other pathogens [36]. These non-specific effects of live vaccines involved the trained immunity which refers to the memory-like characteristics of innate immune cells [37]. Indeed, following exposure to a primary stimulus like a vaccine or a microbial component, innate immune cells, especially monocytes and NK-cells, undergo epigenetic reprogramming that subsequently regulates cytokine production and cell metabolism and it collectively enhances responsiveness to an unrelated secondary stimulus. In this line, observational studies reported a decrease in hospitalization rate and overall mortality among children immunized with live attenuated vaccines [14]. Furthermore, pediatric populations seem to be less vulnerable to COVID-19, especially in low and middle-income countries $[14,38,39]$. The long-term use of an attenuated vaccine, with high coverage rate, could, partially, explain the low symptomatic infection rate among children. Thus, epidemiological studies targeting a largely vaccinated population can help in assessing the protective effect of the MMR vaccine against COVID-19.

\section{Conclusions}

Since December 2019, the novel Coronavirus, SARS-CoV-2, spread all around the word causing a worldwide pandemic, and more than 91 million confirmed cases and a million fatalities.

Using an in silico strategy, this study suggests a possible protective effect of HBV, Tetanus and Measles vaccines against SARS-CoV-2 which should be confirmed by extensive epidemiological studies targeting large populations. This possible cross-protection may explain the variation of the disease severity among countries.

\section{Materials And Methods}

\section{Investigated vaccines and sequences}

Our study focused on twelve vaccines including live attenuated (BCG, OPV, MMR vaccines) and inactivated ones (Tetanus, Corynebacterium diphtheriae, Bordetella pertussis, Hepatitis B, Hepatitis A, Haemophilus influenzae type B (Hib) and Streptococcus pneumoniae vaccines (PCV10) (Table 2).

The full amino-acid sequences of the main antigenic proteins $(n=30)$ corresponding to the 12 investigated vaccines were obtained from NCBI Genbank database (https://www.ncbi.nIm.nih.gov). Accession numbers are listed in Table 2. In addition, the amino-acid sequences of the structural proteins (Spike (S), Envelope (E), Membrane glycoprotein (M), Nucleocapsid (N) and non-structural proteins (ORF1ab, ORF1a, ORF3a, ORF6, ORF7a, ORF7ab, ORF8 and ORF10) of SARS-CoV-2 Wuhan reference strain (NC_045512) were obtained from NCBI.

Table 2: vaccines and corresponding antigenic proteins investigated in this study 


\begin{tabular}{|c|c|c|c|}
\hline Vaccine & Protein & Accession $\mathbf{N}^{\circ}$ & Reference \\
\hline Tetanus & Toxin protein & AAA23282.1 & [40] \\
\hline Corynebacterium diphtheriae & Toxin protein & CAA00374.1 & [40] \\
\hline \multirow[t]{2}{*}{ Hepatitis B } & HBsAg-adw2 & AAW65557.1 & [41] \\
\hline & HBsAg-adr & AAW65588.1 & \\
\hline Bordetella pertussis & Toxin protein & AQW64178.1 & [40] \\
\hline \multirow[t]{2}{*}{ Measles } & Hemagglutinin protein & AAF85705.1 & \\
\hline & Fusion protein & AAF85704.1 & [42] \\
\hline Rubella & Polyprotein E1/E2 & ACN50046.1 & [42] \\
\hline \multirow[t]{2}{*}{ Mumps } & Fusion protein & ACN50030.1 & \\
\hline & Hemagglutinin/neuraminidase protein & ACN50032.1 & [42] \\
\hline \multirow[t]{2}{*}{ Hepatitis A } & VP1 protein & AAA45466.1 & \\
\hline & VP3 protein & AAA45466.1 & {$[43,44]$} \\
\hline \multirow[t]{3}{*}{ Bacillus Calmette-Guérin (BCG) } & Immunogenic protein MPB83 & BAA11027.1 & \\
\hline & Immunogenic protein MPB70 & BAA07402.1 & [45-47] \\
\hline & Immunogenic protein MPB64 & AIC33023.1 & \\
\hline \multirow{2}{*}{$\begin{array}{l}\text { Hemophilus influenzae serotype B } \\
\text { (Hib) }\end{array}$} & Capsulation protein & CWW30252.1 & [40] \\
\hline & Capsular polysaccharide biosynthesis protein & WP_015702013.1 & \\
\hline \multirow[t]{3}{*}{ Poliovirus } & VP1 protein (Sabin 1 strain) & AAL89597.1 & [48] \\
\hline & VP1 protein (Sabin 2 strain) & AAL92486.1 & \\
\hline & VP1 protein (Sabin 3 strain) & AAL89592.1 & \\
\hline \multirow[t]{10}{*}{ Streptococcus pneumoniae (PCV10) } & $\begin{array}{l}\text { Capsular polysaccharide biosynthesis protein [serotype } \\
\text { 19F] }\end{array}$ & AEO88919.1 & [49] \\
\hline & $\begin{array}{l}\text { Capsular polysaccharide biosynthesis protein [serotype } \\
\text { 23F] }\end{array}$ & AAC69522.1 & \\
\hline & $\begin{array}{l}\text { Capsular polysaccharide biosynthesis protein [serotype } \\
\text { 18C] }\end{array}$ & CAI33577.1 & \\
\hline & $\begin{array}{l}\text { Capsular polysaccharide biosynthesis protein [serotype } \\
\text { 14] }\end{array}$ & CAI33319.1 & \\
\hline & $\begin{array}{l}\text { Capsular polysaccharide biosynthesis protein [serotype } \\
\text { 9V] }\end{array}$ & CAI33023.1 & \\
\hline & $\begin{array}{l}\text { Capsular polysaccharide biosynthesis protein [serotype } \\
\text { 7F] }\end{array}$ & CAI32924.1 & \\
\hline & $\begin{array}{l}\text { Capsular polysaccharide biosynthesis protein [serotype } \\
6 \mathrm{~B} \text { ] }\end{array}$ & AAK20683.1 & \\
\hline & $\begin{array}{l}\text { Capsular polysaccharide biosynthesis protein [serotype } \\
\text { 5] }\end{array}$ & CAI32793.1 & \\
\hline & $\begin{array}{l}\text { Capsular polysaccharide biosynthesis protein [serotype } \\
\text { 1] }\end{array}$ & $\cos 99248.1$ & \\
\hline & $\begin{array}{l}\text { Capsular polysaccharide biosynthesis protein [serotype } \\
\text { 4] }\end{array}$ & AAK20668.1 & \\
\hline
\end{tabular}


Identification of similar segments, including identical amino-acids and/or similar amino-acids (with similar biochemical properties), was assessed using Blastp homology search by querying the protein sequences of SARS-CoV-2 over the set of antigenic sequences of the vaccines [50]. Blast 2 sequences tool was used with an Expect threshold (E-value) of 10, in order to see shorter alignments, according to the stochastic model of Karlin and Altschul (1990) [51]. Pairwise alignments obtained from Blastp were explored and analyzed using BioEdit software, version 7.2.5 (http://www.mybiosoftware.com/bioedit-7-0-9-biological-sequence-alignment-editor.html).

\section{Structural analysis and antigenicity prediction}

The structure of the SARS-CoV-2 spike protein was obtained from PDB entries 7BYR [52] and 6LXT [21] corresponding to the structure of $\mathrm{S} 1$ and S2 subunits respectively. Both structures showed a respective sequence identity of 99.6 and $100 \%$ compared to the reference sequence of the S protein from the Wuhan-Hu-1 isolate of SARS-CoV-2 (accession number YP_009724390.1 for the spike protein). The segments matching one of the sequences of $S$ and $N$ proteins were mapped on the structure. The Solvent Accessible Surface Area (SASA) per residue was calculated using freesasa [52]. The B-cell and T-cell epitope predictions were conducted using IEDB analysis resource Bepipred 2.0 [29] and the IEDB analysis resource NetMHCpan [53] methods by uploading the primary structure of SARS-CoV-2 protein; considering all the possible human HLA alleles for MHC class I. These correspond to HLA genes A, B, C, E, and G and cover 134 alleles from different allele groups. A list of these alleles is provided in Supplementary Material 15. The length of the predicted peptides was set to a default value of 8-11 residues, with respect to the proteasomal processing mechanism [54]. A pattern is retained if it shows a good quality local alignment with no indels and no more than two successive dissimilar residues. The matching pattern of the query has to show significant antigenicity prediction, at least with one of the methods, IEDB Bepipred or IEDB NetMHCpan. A cutoff of peptide score no less than 0.1 was used. At this level, the sensitivity and specificity values would be above 0.9 , according to the evaluation by Jutz et al [55]. For IEDB Bepipred, a putative epitope has to show a score above 0.5 for all its constructing amino acids.

The Solvent Accessible Surface Area (SASA) was calculated residue wise. Three probing radii were used including one that mimics the solvent molecules $(1.4 \AA$ ) and two other ( 5 and $10 \AA$ ) to access the accessibility of the Antibody Complementarity-Determining region (CDR) to the putative B-cell epitope [56].

\section{Declarations}

Ethics approval and consent to participate: This study did not include Human participants or Patient data. Hence no ethical approval and consent to participate is required.

Consent for publication: Not applicable. This study did not include patients.

Availability of data and materials: All data generated or analyzed as part of this study are included in this published article and its supplementary information files. Accession numbers of sequences used in this study are indicated in Table2, in the Material and Methods section of the article. All data generated are available in a public repository https://figshare.com/articles/dataset/Comparative_study_of_SARS-CoV-

2_proteins_and_antigenic_proteins_in_BCG_OPV_MMR_and_other_vaccines_evidence_of_possible_putative_protective_effect/13220762

Competing interests: The authors declare that they have no competing interests.

Funding: This study was funded by the Tunisian Ministry of Higher Education and Scientific Research (Research laboratory: Virus, Vectors and Hosts; LR20IPT10). It was also partially supported by the European project PHINDaccess: Strengthening Omics data analysis capacities in pathogen-host interaction (Grant agreement ID: 811034).

Authors' contributions: SH-B, HO and KG designed the study, SH-B and HO wrote the main text, HO, RT, KA and ML contributed to carry out analysis and to prepare figures, SH-B, IBM, MK and HT validated the study. All authors read and approved the final manuscript.

Acknowledgment: This study was funded by the Tunisian ministry of higher education and Scientific Research (Research laboratory: Virus, Vectors and Hosts). It was also partially supported by the European project PHINDaccess: Strengthening Omics data analysis capacities in pathogen-host interaction (Grant agreement ID: 811034). 
S Haddad-Boubaker, PhD, HDR in Virology from the Faculty of Sciences of Tunis. She is an Assistant Professor in the Laboratory of Clinical Virology, at Pasteur Institute of Tunis, which acts as the WHO Regional Reference Laboratory for Poliomyelitis and Measles in the EMR. She is also a Professor of Clinical Virology and the coordinator of the Microbiology section in the High Institute of Health Techniques in Tunis, Tunisia. Her research interest includes molecular characterization and omics data analysis of Human viruses, especially poliovirus, enteric viruses and SARS-CoV2.

H Othman, $\mathrm{PhD}$ is a bioinformatician at the Sydney Brenner Institute for Molecular Bioscience at the University of the Witwatersrand. His main interests are pharmacogenomics, Molecular modeling, and data science. He is an avid supporter of reproducible research practices and data sharing trying to increase awareness about these issues in bioinformatics and genomics fields.

R Touati, PhD in electrical engineering from the National Engineering School of Tunisia (ENIT). Currently, she has a Postdoctoral position at the Laboratory of Human Genetics (LR99ES10) at the Faculty of Medicine of Tunis (FMT). Her research interest includes genomic signal processing, bioinformatics, pattern recognition and machine learning.

K Ayouni, PhD student in the Laboratory of Clinical Virology, at Pasteur Institute of Tunis, Tunisia.

M Lakhal, PhD student in Human Genetics at the Faculty of Medicine of Tunis, Tunisia.

Prof. I Ben Mustapha, MD. She is a Professor of Immunology at the Faculty of Medicine of Tunis and the head of a research team at the Laboratory of Transmission, Control and Immunobiology of Infections at Institut Pasteur de Tunis, Tunisia.

K Ghedira, PhD. He is an assistant professor in Bioinformatics at the Institut Pasteur de Tunis. He is head of the research team at the Laboratory of Biomathematics, Biomathematics and Biostatistics in Institut Pasteur de Tunis, Tunisia. His research interests are mainly focused on OMICS data integration and functional genomics.

Prof. M Kharrat, PhD in Human Genetics from the Faculty of Medicine of Tunis (FMT). He is a Professor of Human Genetics in 2006 at the Faculty of Medicine of Tunis (FMT). He is the head of the Genetic Human laboratory (LR99ES10) at the Faculty of Medicine of Tunis (FMT). His research interests include Human Genetics.

Prof. H Triki, MD is Professor in Virology since 2006 at the Faculty of Medicine of Tunis (FMT). She is the head of the Laboratory of Clinical Virology, which acts as the WHO Regional Reference Laboratory for Poliomyelitis and Measles in the EMR region. In 2018, she was assigned as Director of the Clinical Investigation Center entitled: "Transmissible diseases: Natural history and innovative tools for diagnostic, prevention and treatment" in Pasteur Institute of Tunis. Currently, she is a member of the National COVID-19 vaccination comity.

\section{References}

1. Li G, De Clercq E. Therapeutic options for the 2019 novel coronavirus (2019-nCoV). Nature reviews Drug discovery. 2020;19(3):14950.

2. WHO. WHO Coronavirus Disease (COVID-19) Dashboard 2020 [15 January 2020]. Available from: https://covid19.who.int/.

3. Coronaviridae Study Group of the International Committee on Taxonomy of V. The species Severe acute respiratory syndromerelated coronavirus: classifying 2019-nCoV and naming it SARS-CoV-2. Nature microbiology. 2020;5(4):536-44.

4. Touati R, Haddad-Boubaker S, Ferchichi I, Messaoudi I, Ouesleti AE, Triki H, et al. Comparative genomic signature representations of the emerging COVID-19 coronavirus and other coronaviruses: High identity and possible recombination between Bat and Pangolin coronaviruses. Genomics. 2020.

5. Wu F, Zhao S, Yu B, Chen YM, Wang W, Song ZG, et al. A new coronavirus associated with human respiratory disease in China. Nature. 2020;579(7798):265-9.

6. WHO. Coronavirus disease (COVID-19) Weekly Epidemiological Update 2020 [22 October 2020]. Available from: https://www.who.int/emergencies/diseases/novel-coronavirus-2019/situation-reports.

7. Hallal PC, Hartwig FP, Horta BL, Silveira MF, Struchiner CJ, Vidaletti LP, et al. SARS-CoV-2 antibody prevalence in Brazil: results from two successive nationwide serological household surveys. The Lancet Global health. 2020;8(11):e1390-e8. 
8. Lauxmann MA, Santucci NE, Autran-Gomez AM. The SARS-CoV-2 Coronavirus and the COVID-19 Outbreak. International braz j urol : official journal of the Brazilian Society of Urology. 2020;46(suppl.1):6-18.

9. Martines RB, Ritter JM, Matkovic E, Gary J, Bollweg BC, Bullock H, et al. Pathology and Pathogenesis of SARS-CoV-2 Associated with Fatal Coronavirus Disease, United States. Emerging infectious diseases. 2020;26(9):2005-15.

10. Haider N, Osman AY, Gadzekpo A, Akipede GO, Asogun D, Ansumana R, et al. Lockdown measures in response to COVID-19 in nine sub-Saharan African countries. BMJ global health. 2020;5(10).

11. Post LA, Argaw ST, Jones C, Moss CB, Resnick D, Singh LN, et al. A SARS-CoV-2 Surveillance System in Sub-Saharan Africa: Modeling Study for Persistence and Transmission to Inform Policy. Journal of medical Internet research. 2020;22(11):e24248.

12. Riggioni $\mathrm{C}$, Comberiati P, Giovannini M, Agache I, Akdis M, Alves-Correia M, et al. A compendium answering 150 questions on COVID19 and SARS-CoV-2. Allergy. 2020;75(10):2503-41.

13. Curtis N, Sparrow A, Ghebreyesus TA, Netea MG. Considering BCG vaccination to reduce the impact of COVID-19. Lancet. 2020;395(10236):1545-6.

14. O'Neill LAJ, Netea MG. BCG-induced trained immunity: can it offer protection against COVID-19? Nature reviews Immunology. 2020;20(6):335-7.

15. Vojtek I, Buchy P, Doherty TM, Hoet B. Would immunization be the same without cross-reactivity? Vaccine. 2019;37(4):539-49.

16. Hamiel U, Kozer E, Youngster I. SARS-CoV-2 Rates in BCG-Vaccinated and Unvaccinated Young Adults. Jama. 2020.

17. Escobar LE, Molina-Cruz A, Barillas-Mury C. BCG vaccine protection from severe coronavirus disease 2019 (COVID-19). Proceedings of the National Academy of Sciences of the United States of America. 2020;117(30):17720-6.

18. Sidiq KR, Sabir DK, Ali SM, Kodzius R. Does Early Childhood Vaccination Protect Against COVID-19? Frontiers in molecular biosciences. 2020;7:120.

19. He Y, Li J, Heck S, Lustigman S, Jiang S. Antigenic and immunogenic characterization of recombinant baculovirus-expressed severe acute respiratory syndrome coronavirus spike protein: implication for vaccine design. Journal of virology. 2006;80(12):5757-67.

20. Zhang BZ, Hu YF, Chen LL, Yau T, Tong YG, Hu JC, et al. Mining of epitopes on spike protein of SARS-CoV-2 from COVID-19 patients. Cell research. 2020;30(8):702-4.

21. Xia S, Liu M, Wang C, Xu W, Lan Q, Feng S, et al. Inhibition of SARS-CoV-2 (previously 2019-nCoV) infection by a highly potent pancoronavirus fusion inhibitor targeting its spike protein that harbors a high capacity to mediate membrane fusion. Cell research. 2020;30(4):343-55.

22. Hashiguchi T, Kajikawa M, Maita N, Takeda M, Kuroki K, Sasaki K, et al. Crystal structure of measles virus hemagglutinin provides insight into effective vaccines. Proceedings of the National Academy of Sciences of the United States of America. 2007;104(49):19535-40.

23. Dougan DA, Malby RL, Gruen LC, Kortt AA, Hudson PJ. Effects of substitutions in the binding surface of an antibody on antigen affinity. Protein engineering. 1998;11(1):65-74.

24. Frankild S, de Boer RJ, Lund O, Nielsen M, Kesmir C. Amino acid similarity accounts for T cell cross-reactivity and for "holes" in the T cell repertoire. PloS one. 2008;3(3):e1831.

25. Singh H, Ansari HR, Raghava GP. Improved method for linear B-cell epitope prediction using antigen's primary sequence. PloS one. 2013;8(5):e62216.

26. Tajiri K, Ozawa T, Jin A, Tokimitsu Y, Minemura M, Kishi H, et al. Analysis of the epitope and neutralizing capacity of human monoclonal antibodies induced by hepatitis B vaccine. Antiviral research. 2010;87(1):40-9.

27. Lu LL, Suscovich TJ, Fortune SM, Alter G. Beyond binding: antibody effector functions in infectious diseases. Nature reviews Immunology. 2018;18(1):46-61.

28. Arvin AM, Fink K, Schmid MA, Cathcart A, Spreafico R, Havenar-Daughton C, et al. A perspective on potential antibody-dependent enhancement of SARS-CoV-2. Nature. 2020;584(7821):353-63.

29. Baughn LB, Sharma N, Elhaik E, Sekulic A, Bryce AH, Fonseca R. Targeting TMPRSS2 in SARS-CoV-2 Infection. Mayo Clinic proceedings. 2020;95(9):1989-99.

30. Poh CM, Carissimo G, Wang B, Amrun SN, Lee CY, Chee RS, et al. Two linear epitopes on the SARS-CoV-2 spike protein that elicit neutralising antibodies in COVID-19 patients. Nature communications. 2020;11(1):2806.

31. Cagliani R, Forni D, Clerici M, Sironi M. Coding potential and sequence conservation of SARS-CoV-2 and related animal viruses. Infection, genetics and evolution : journal of molecular epidemiology and evolutionary genetics in infectious diseases. 
2020;83:104353.

32. Trigueiro-Louro J, Correia V, Figueiredo-Nunes I, Giria M, Rebelo-de-Andrade H. Unlocking COVID therapeutic targets: A structurebased rationale against SARS-CoV-2, SARS-CoV and MERS-CoV Spike. Computational and structural biotechnology journal. 2020;18:2117-31.

33. Walls AC, Park YJ, Tortorici MA, Wall A, McGuire AT, Veesler D. Structure, Function, and Antigenicity of the SARS-CoV-2 Spike Glycoprotein. Cell. 2020;181(2):281-92 e6.

34. He Y, Zhou Y, Wu H, Luo B, Chen J, Li W, et al. Identification of immunodominant sites on the spike protein of severe acute respiratory syndrome (SARS) coronavirus: implication for developing SARS diagnostics and vaccines. Journal of immunology. 2004;173(6):4050-7.

35. Schaecher SR, Mackenzie JM, Pekosz A. The ORF7b protein of severe acute respiratory syndrome coronavirus (SARS-CoV) is expressed in virus-infected cells and incorporated into SARS-CoV particles. Journal of virology. 2007;81(2):718-31.

36. Uthayakumar D, Paris S, Chapat L, Freyburger L, Poulet H, De Luca K. Non-specific Effects of Vaccines Illustrated Through the BCG Example: From Observations to Demonstrations. Frontiers in immunology. 2018;9:2869.

37. Netea MG, Dominguez-Andres J, Barreiro LB, Chavakis T, Divangahi M, Fuchs E, et al. Defining trained immunity and its role in health and disease. Nature reviews Immunology. 2020;20(6):375-88.

38. Patel NA. Pediatric COVID-19: Systematic review of the literature. American journal of otolaryngology. 2020;41(5):102573.

39. Yoldas MA, Yoldas H. Pediatric COVID-19 Disease: A Review of the Recent Literature. Pediatric annals. 2020;49(7):e319-e25.

40. Dhillon S. DTPa-HBV-IPV/Hib Vaccine (Infanrix hexa): A Review of its Use as Primary and Booster Vaccination. Drugs. 2010;70(8):1021-58.

41. Heijtink RA, Bergen P, Melber K, Janowicz ZA, Osterhaus AD. Hepatitis B surface antigen (HBsAg) derived from yeast cells (Hansenula polymorpha) used to establish an influence of antigenic subtype (adw2, adr, ayw3) in measuring the immune response after vaccination. Vaccine. 2002;20(17-18):2191-6.

42. Tillieux SL, Halsey WS, Sathe GM, Vassilev V. Comparative analysis of the complete nucleotide sequences of measles, mumps, and rubella strain genomes contained in Priorix-Tetra and ProQuad live attenuated combined vaccines. Vaccine. 2009;27(16):2265-73.

43. Haro I, Perez S, Garcia M, Chan WC, Ercilla G. Liposome entrapment and immunogenic studies of a synthetic lipophilic multiple antigenic peptide bearing VP1 and VP3 domains of the hepatitis A virus: a robust method for vaccine design. FEBS letters. 2003;540(1-3):133-40.

44. Ping LH, Jansen RW, Stapleton JT, Cohen JI, Lemon SM. Identification of an immunodominant antigenic site involving the capsid protein VP3 of hepatitis A virus. Proceedings of the National Academy of Sciences of the United States of America. 1988;85(21):8281-5.

45. Harboe M, Nagai S, Patarroyo ME, Torres ML, Ramirez C, Cruz N. Properties of proteins MPB64, MPB70, and MPB80 of Mycobacterium bovis BCG. Infection and immunity. 1986;52(1):293-302.

46. Seki M, Honda I, Fujita I, Yano I, Yamamoto S, Koyama A. Whole genome sequence analysis of Mycobacterium bovis bacillus Calmette-Guerin (BCG) Tokyo 172: a comparative study of BCG vaccine substrains. Vaccine. 2009;27(11):1710-6.

47. Wiker HG, Nagai S, Hewinson RG, Russell WP, Harboe M. Heterogenous expression of the related MPB70 and MPB83 proteins distinguish various substrains of Mycobacterium bovis BCG and Mycobacterium tuberculosis H37Rv. Scandinavian journal of immunology. 1996;43(4):374-80.

48. Katz SL. From culture to vaccine-Salk and Sabin. The New England journal of medicine. 2004;351(15):1485-7.

49. Croxtall JD, Keating GM. Pneumococcal polysaccharide protein D-conjugate vaccine (Synflorix; PHiD-CV). Paediatric drugs. 2009;11(5):349-57.

50. Altschul SF, Gish W, Miller W, Myers EW, Lipman DJ. Basic local alignment search tool. Journal of molecular biology. 1990;215(3):403-10.

51. Karlin S, Altschul SF. Methods for assessing the statistical significance of molecular sequence features by using general scoring schemes. Proceedings of the National Academy of Sciences of the United States of America. 1990;87(6):2264-8.

52. Cao Y, Su B, Guo X, Sun W, Deng Y, Bao L, et al. Potent Neutralizing Antibodies against SARS-CoV-2 Identified by High-Throughput Single-Cell Sequencing of Convalescent Patients' B Cells. Cell. 2020;182(1):73-84 e16.

53. Reynisson B, Alvarez B, Paul S, Peters B, Nielsen M. NetMHCpan-4.1 and NetMHCIlpan-4.0: improved predictions of MHC antigen presentation by concurrent motif deconvolution and integration of MS MHC eluted ligand data. Nucleic acids research.

Page 11/13 
2020;48(W1):W449-W54.

54. Kisselev AF, Akopian TN, Woo KM, Goldberg AL. The sizes of peptides generated from protein by mammalian 26 and 20 S proteasomes. Implications for understanding the degradative mechanism and antigen presentation. The Journal of biological chemistry. 1999;274(6):3363-71.

55. Jurtz V, Paul S, Andreatta M, Marcatili P, Peters B, Nielsen M. NetMHCpan-4.0: Improved Peptide-MHC Class I Interaction Predictions Integrating Eluted Ligand and Peptide Binding Affinity Data. Journal of immunology. 2017;199(9):3360-8.

56. Urbanowicz RA, Wang R, Schiel JE, Keck ZY, Kerzic MC, Lau P, et al. Antigenicity and Immunogenicity of Differentially Glycosylated Hepatitis C Virus E2 Envelope Proteins Expressed in Mammalian and Insect Cells. Journal of virology. 2019;93(7).

57. Fremont DH, Matsumura M, Stura EA, Peterson PA, Wilson IA. Crystal structures of two viral peptides in complex with murine MHC class I H-2Kb. Science. 1992;257(5072):919-27.

\section{Figures}

(A)

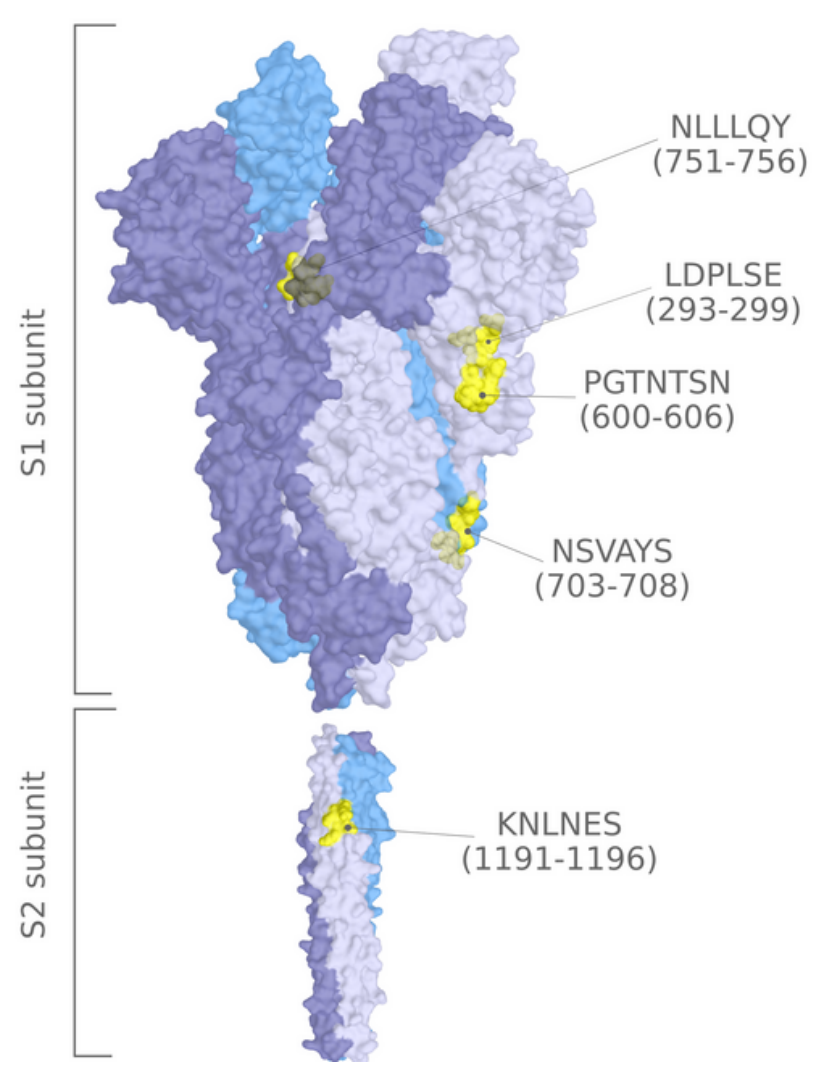

(B)

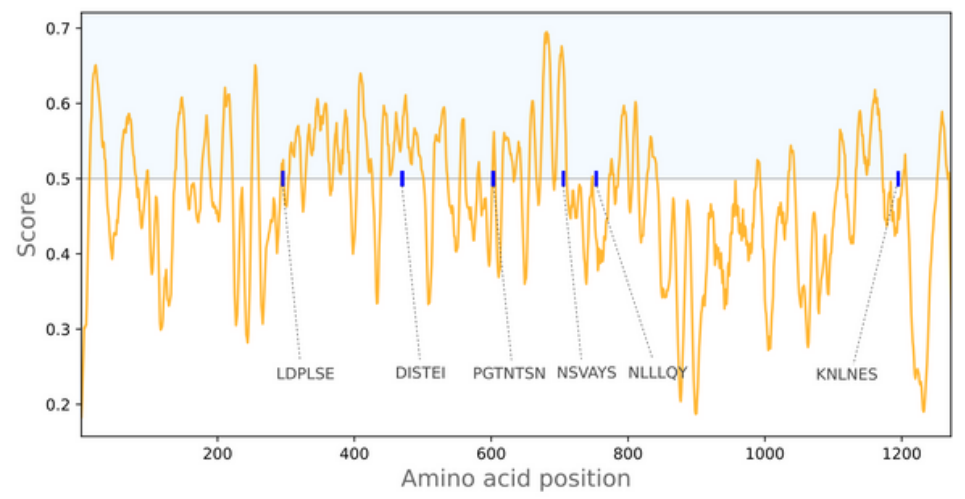

(C)

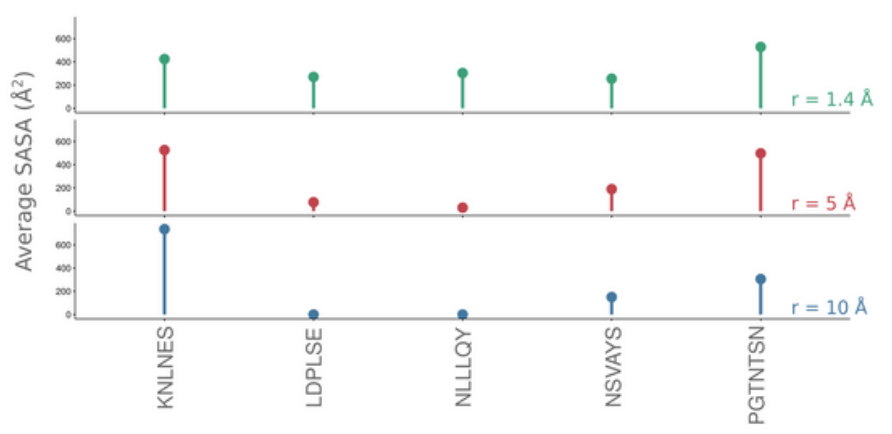

\section{Figure 1}

Structural mapping in S protein of the segments that match the antigenic proteins from different pathogens. (A) The location of the segments on the structure is marked by yellow patches. Different chains are represented in different colors. The S1 and S2 subunits have been solved independently. (B) B-cell epitope prediction from the sequence of SARS-CoV-2 protein. The sequences identified from the similarity analysis are marked in blue. Segments in which amino acid scores are above 0.5 are putative epitope sites. (C) Cumulative SASA measures for each of the putative antigenic sites calculated using different probe radii. 
(A)

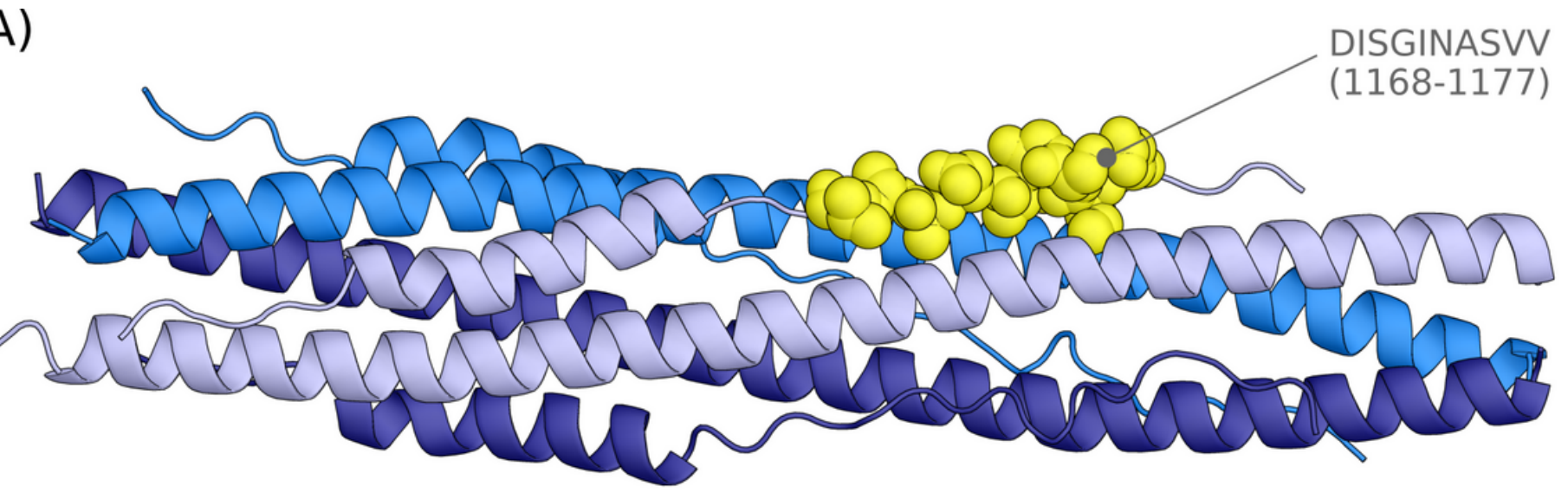

(B)

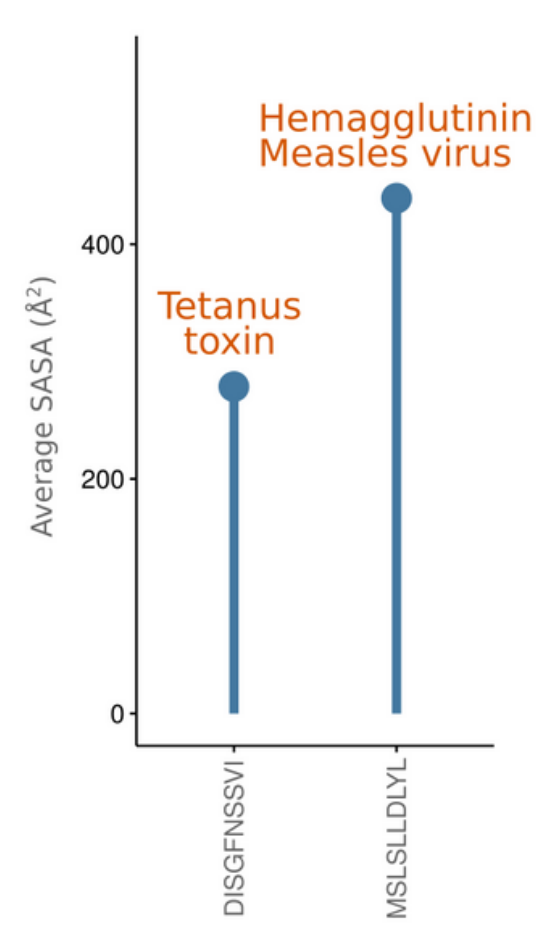

(C)

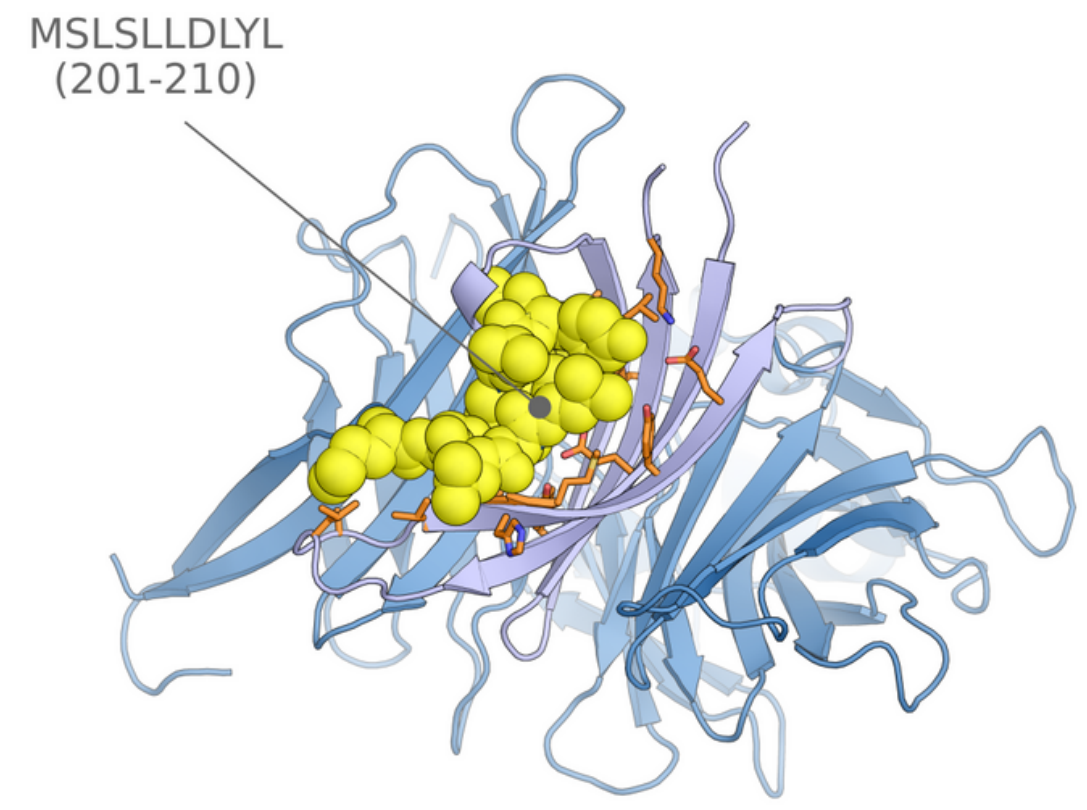

Figure 2

Structural properties of $\mathrm{CMH}-\mathrm{I}$ putative epitopes resembling segments from Tetanus toxin protein and chain A of the Measles hemagglutinin Protein. (A) Location of DISGINASVV on the structure of the six-helical bundle fusion core from the spike protein. (B) Calculation of SASA for the vaccines DISGFNSSVI and MSLSLLDLYL segment using a probe of $1.4 \AA$. (C) Crystal structure of the Measles virus hemagglutinin [22]. The peptide (in yellow) shows putative T-cell immunogenicity with the interaction pocket residues (light purple).

\section{Supplementary Files}

This is a list of supplementary files associated with this preprint. Click to download.

- Supplementarymaterial1.docx

- Additionalmat2130711.docx

- Supplementarymaterial14.docx

- supplementarymaterial15.docx 